\title{
Pyogenic granuloma under nail: A rare localization
}

\section{Aida Oulehri, Zakia Douhi, Kaoutar Acheboune, Hanane Baybay, Sara Elloudi, Fatima Zahra Mernissi}

Dermatology Department of the University Hospital Center Hassan II, Fez, Morocco

Corresponding author: Aida Oulehri, MD, E-mail: aidaoulehri@gmail.com

Pyogenic granuloma (PG) - also known as lobular capillary hemangioma - is a benign vascular tumour that occurs on the skin and mucous membranes, it's can arise spontaneously, in sites of injury, or within capillary malformations [1]. Because malignant tumors, such as amelanotic melanoma, can be misdiagnosed as PG, histopathology is mandatory for the correct diagnosis, even though dermoscopy can play a primary role in the prompt recognition of PG, showing highly repetitive patterns that can support the diagnosis [2]. In particular, the presence of reddish homogeneous area, white rail lines are strongly suggestive of PG; even more so when associated together. On the other hand, with dermoscopy, it is not uncommon to detect ulceration and vascular structures that do not rule out malignancies [3]. Vascular structures are observed in $45 \%$ of cases, they can be linear-irregular or polymorphic and atypical [2]. Despite its benign nature, GP can have a significant negative impact on patients' quality of life, and therefore appropriate therapy is needed.

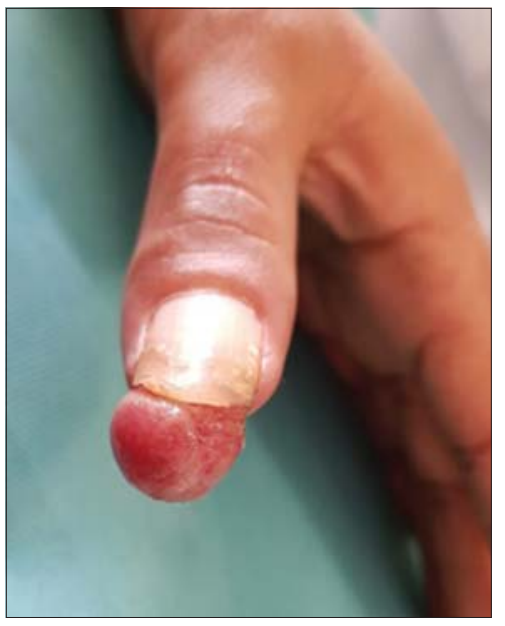

Figure 1: Under ungueal botryomycoma.
This is a 35-year-old patient with a history of trauma to his left hand dating back 3 months before his admission. He presents with a nodule under his left

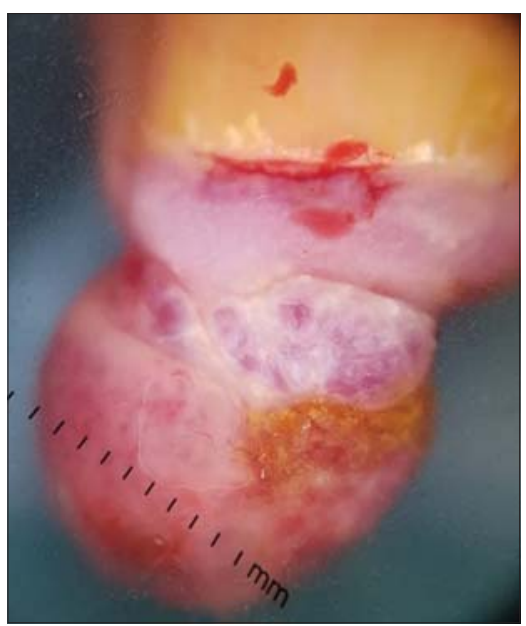

Figure 2: Presence on dermatoscopy of lagoon with septa fibrous and linear vessels.

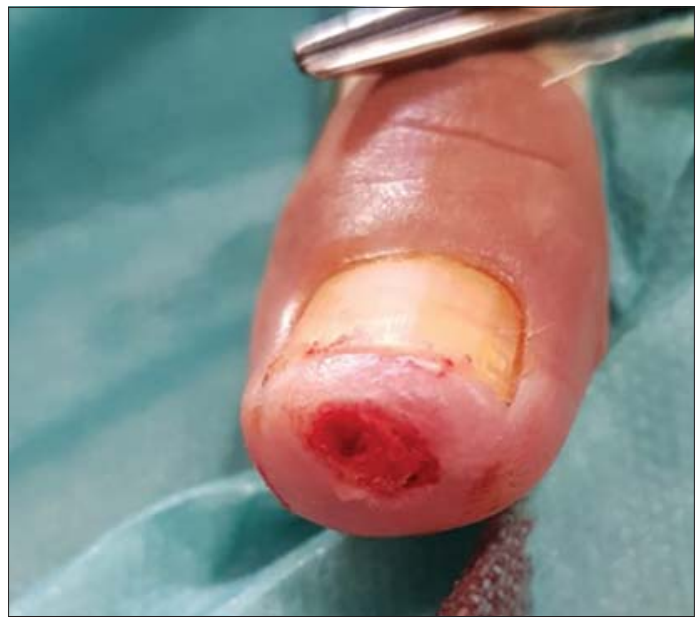

Figure 3: Surgical excision of the pyogenic granuloma.

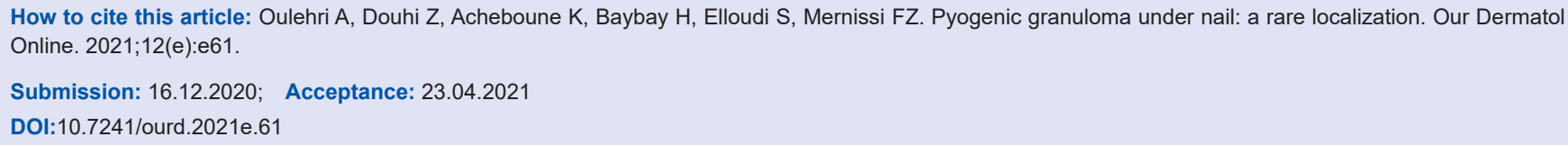


thumb, which has been evolving for 2 months, fixed in depth and sometimes-bleeding (Fig. 1). The nodule was slightly painful. X-rays of the thumb were normal. On dermoscopy (Fig. 2), we noted the presence of vascular lagoons with fibrous septal and linear vessels. We suspected a botryomyoma or achromic melanoma. Surgical excision was performed (Fig. 3) with an anatomopathological study in favour of a pyogenic granuloma.

\section{Consent}

The examination of the patient was conducted according to the principles of the Declaration of Helsinki.

The authors certify that they have obtained all appropriate patient consent forms, in which the patients gave their consent for images and other clinical information to be included in the journal. The patients understand that their names and initials will not be published and due effort will be made to conceal their identity, but that anonymity cannot be guaranteed.

\section{REFERENCES}

1. Wollina U, Langner D, França K, Gianfaldoni S, Lotti T, Tchernev G. Pyogenic granuloma - a common benign vascular tumor with variable clinical presentation: new findings and treatment options. Open Access Maced J Med Sci. 2017;5:423-6.

2. Zaballos P, Carulla M, Ozdemir F, Zalaudek I, Bañuls J, Llambrich A, et al. Dermoscopy of pyogenic granuloma: a morphological study. Br J Dermatol. 2010;163:1229-37.

3. Piccolo V, Russo T, Moscarella E, Brancaccio G, Alfano R, Argenziano G. Dermatoscopy of vascular lesions. Dermatol Clin. 2018;36:389-95.

Copyright by Aida Oulehri, et al. This is an open-access article distributed under the terms of the Creative Commons Attribution License, which permits unrestricted use, distribution, and reproduction in any medium, provided the original author and source are credited.

Source of Support: Nil, Conflict of Interest: None declared. 